\title{
A NEW IMPROVED SCHEME FOR PARTIAL DIFFERENTIAL EQUATIONS WITH VARIABLE COEFFICIENTS IN SEMI-INFINITE DOMAIN VIA DOUBLE RATIONAL CHEBYSHEV FUNCTIONS
}

\author{
Mahmoud A. Nassar ${ }^{1}$, Kamal R. Raslan ${ }^{2}$ and Mohamed A. Ramadan ${ }^{3}$ \\ ${ }^{1,2}$ Mathematics Department, Faculty of Science, Al-Azhar University, Nasr-City, Cairo, Egypt. \\ ${ }^{3}$ Faculty of Science, Menoufia University, Shebein El-Koom, Egypt. \\ Email addresses: \\ ${ }^{1}$ m7moudscience@yahoo.com \\ ${ }^{2}$ kamal_raslan@yahoo.com \\ ${ }^{3}$ mramadan@eun.eg; ramadanmohamed13@yahoo.com;
}

\begin{abstract}
The concept of double rational Chebyshev functions on the semi-infinite domain $(0 \leq x, y<\infty)$ and some of their properties are introduced in this work. Also, the definition of derivatives for double rational Chebyshev functions is improved. This new definition is employed to deal with partial differential equations with variable coefficients derived on the interval $[0, \infty)$. The new definition with the spectral collocation method generates a new improved scheme. Numerical results are show that demonstrates the validity and applicability of the two techniques. The obtained numerical results are compared with the exact solution where it shown to be very attractive with good accuracy.
\end{abstract}

Keywords: Double rational Chebyshev (DRC) functions, partial differential equations (PDEs), semiinfinite domain, collocation method.

\section{INTRODUCTION}

The partial differential equations (PDEs) have an important role owing to their applications in real life phenomena and applied mathematics for instance, the wave equation, heat, Laplace, Helmholtz, Klein-Gordon, and Poisson's equations. The spectral methods are one of the most widely used methods to solve PDEs. Chebyshev polynomials are one of the most wellknown of those special functions. Chebyshev polynomials are orthogonal in the domain $(-1 \leq x \leq 1)$ with respect to the weight function $w(x)=\frac{1}{\sqrt{1-x^{2}}}$ and can be written the recurrence formulae as:

$$
T_{0}(x)=1, \quad T_{1}(x)=x, \quad T_{n+1}(x)=2 x T_{n}(x)-T_{n-1}(x), \quad n \geq 1 .
$$

As a notation, Chebyshev polynomials are defined in a closed interval $(-1 \leq x \leq 1)$ in one variable [1-6] but many of studies have worked to extended to multi-variable cases, especially in two variables [7] and [8]. It is clear that if Chebyshev approach deals with problems defined on larger domains, especially, when it has unbounded domain, it causes a failure and weak approximation. For this it is more suitable to generate a new set of basis for the interval $[0, \infty)$ using a transformation that maps a finite domain into an semi-infinite interval this idea is introduced by Boyed in 1987 [9] where the new basis will get most of the good numerical characteristics of the Chebyshev polynomials called Rational Chebyshev (RC) functions that are orthogonal in $L_{2}(0, \infty)$. Ramadan et al. [10-17], Parand and Razzaghi [18], Parand et al. [19], and Sezer et al. [20] are used RC functions to solve differential equations and its applications.

All previous works and studies carried out for single variable using RC functions, we study the definition of RC functions in two-variables. In this paper, the definition of double rational Chebyshev (DRC) functions on the semi-infinite domain $(0 \leq x, y<\infty)$, and some properties of DRC functions 
are obtained. Also, the derivatives of RC functions in two variables are improved in this work. The DRC collocation technique is used for solving PDEs defined on the semi-infinite domains.

\section{Double rational Chebyshev functions[8] and [22]}

The RC functions $R_{n}(x)$ of the first kind are functions in one variable $x$ of degree $n$, defined by the relation

$$
R_{n}(x)=T_{n}\left(\frac{x-1}{x+1}\right), \quad \text { when } \quad x=\cot ^{2}(\theta / 2), x \in[0, \infty)
$$

The DRC functions as:

$$
R_{m, n}(x, y)=R_{m}(x) R_{n}(y), \quad \text { where } \quad R_{m}(x)=T_{m}\left(\frac{x-1}{x+1}\right), R_{n}(y)=T_{n}\left(\frac{y-1}{y+1}\right) .
$$

The recurrence relation for DRC functions satisfies:

$$
\begin{aligned}
& R_{r+1, s}(x, y)=\left[2 R_{1}(x) R_{r}(x)-R_{r-1}(x)\right] R_{s}(y), \\
& R_{r, s+1}(x, y)=R_{r}(x)\left[2 R_{1}(y) R_{s}(y)-R_{s-1}(y)\right] .
\end{aligned}
$$

The weight function in the double form is:

$$
w(x, y)=\frac{(x y)^{-\frac{1}{2}}}{(x+1)(y+1)},
$$

It is the proper for choice for the DRC functions to be orthogonal, with the orthogonality condition

$$
\int_{0}^{\infty} \int_{0}^{\infty} R_{i, j}(x, y) R_{k, l}(x, y) w(x, y) d x d y=\left\{\begin{array}{ll}
\pi^{2} & i=j=k=l=0 \\
\frac{\pi^{2}}{4} & i=k \neq 0, j=l \neq 0 \\
\frac{\pi^{2}}{2} & i=k=0, j=l \neq 0 \\
& \text { or } i=k \neq 0, j=l=0 \\
0 & \text { otherwise }
\end{array} .\right.
$$

The following relation is known as the product relation of DRC functions which can be represented as:

$$
R_{m, n}(x, y) \cdot R_{i, j}(x, y)=\frac{1}{4}\left[R_{m+i, n+j}(x, y)+R_{m+i,|n-j|}(x, y)+R_{|m-i|, n+j}(x, y)+R_{|m-i|,|n-j|}(x, y)\right] .
$$

\section{The partial derivatives of DRC functions [21]}

In the next propositions we improve the concept of partial derivatives of DRC functions in terms of itself.

\section{Proposition 3.1}

The derivative of DRC functions of order $(r, s)$ th is given in terms of itself by the relation

$$
\mathbf{R}^{(r, s)}(x, y) \cong \mathbf{R}(x, y)\left(\mathbf{D}_{x}\right)^{r}\left(\mathbf{D}_{y}\right)^{s},
$$

where, $\mathbf{D}_{x}$ and $\mathbf{D}_{y}$ are $(i+1)(j+1) \times(i+1)(j+1)$ which can be obtained by $\mathbf{D}_{x}=\mathbf{D}_{1}+\mathbf{D}_{2}$, where 


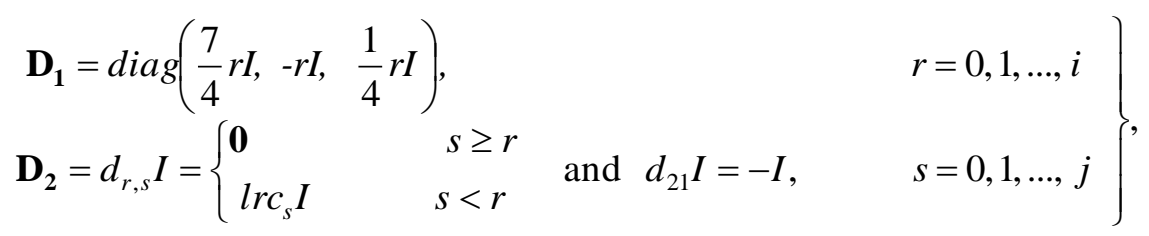

where $l=(-1)^{r+s+1}, c_{1}=1$ and $c_{s}=2$ for $s \geq 2$,

and

$$
\mathbf{D}_{y}=\left[\begin{array}{cccc}
\omega & 0 & \cdots & 0 \\
0 & \omega & \cdots & 0 \\
\vdots & \vdots & \ddots & \vdots \\
0 & 0 & \cdots & \omega
\end{array}\right]^{T}, \omega=S_{1}+S_{2}, \text { and } s_{21}=-1
$$

where,

$$
S_{1}=\operatorname{diag}\left(\frac{7}{4} s,-s, \frac{1}{4} s\right), s=0,1,2, \ldots, i
$$

and the components of $S_{2}$ are $s_{k l}$ obtained from:

$$
s_{k l}=\left\{\begin{array}{lc}
0, & l \geq k \\
l(r) c_{s}, & l<k
\end{array},\right.
$$

where $I$ is identity matrix, 0 is zero matrix and $\omega$ is square matrix. The dimensions for these three matrices are $(j+1) \times(j+1)$ which are components of the matrices $\mathbf{D}_{x}$ and $\mathbf{D}_{y}$.

\section{Proof:}

By using recurrence relation (2.3) we can demonstrate the partial derivatives of the DRC functions, first dealing with the variable $x$, and by using the multiplication relation we get

$$
\begin{gathered}
\frac{\partial}{\partial x}\left(R_{0, n}(x, y)\right)=0, \text { for all } n, \\
\frac{\partial}{\partial x}\left(R_{1, n}(x, y)\right)=\frac{4}{(1+x)^{2}} R_{n}(y)=\left(\frac{3}{4} R_{0}(x)-R_{1}(x)+\frac{1}{4} R_{2}(x)\right) R_{n}(y), \\
=\frac{3}{4} R_{0, n}(x, y)-R_{1, n}(x, y)+\frac{1}{4} R_{2, n}(x, y),
\end{gathered}
$$

and

$$
\begin{aligned}
\frac{\partial}{\partial x}\left(R_{m+1, n}(x, y)\right) & =\frac{\partial}{\partial x}\left[2 R_{1}(x) R_{m, n}(x, y)-R_{m-1, n}(x, y)\right] \\
& =\frac{\partial}{\partial x}\left[2\left(R_{1}(x)\right)^{(0,0)}\left(R_{m, n}(x, y)\right)^{(0,0)}-\left(R_{m-1, n}(x, y)\right)^{(0,0)}\right] \\
& =\left[2\left(R_{1}(x)\right)^{(1,0)}\left(R_{m, n}(x, y)\right)^{(0,0)}+2\left(R_{1}(x)\right)^{(0,0)}\left(R_{m, n}(x, y)\right)^{(1,0)}-\left(R_{m-1, n}(x, y)\right)^{(1,0)}\right] .
\end{aligned}
$$

Using the relations (3.4), (3.5) and (3.6) and by using multiplication relation (2.4) for $m=0,1, \ldots$, $i$, the components of the block matrix of derivatives $\mathbf{D}_{x}$ can be shown as the following: 


$$
\left\{\begin{array}{l}
\left(R_{0, n}(x, y)\right)^{(1,0)}=0, \\
\left(R_{1, n}(x, y)\right)^{(1,0)}=\frac{3}{4} R_{0, n}(x, y)-R_{1, n}(x, y)+\frac{1}{4} R_{2, n}(x, y), \\
\left(R_{2, n}(x, y)\right)^{(1,0)}=-2 R_{0, n}(x, y)+\frac{7}{2} R_{1, n}(x, y)-2 R_{2, n}(x, y)+\frac{1}{2} R_{3, n}(x, y), \\
\left(R_{3, n}(x, y)\right)^{(1,0)}=3 R_{0, n}(x, y)-6 R_{1, n}(x, y)+\frac{21}{4} R_{2, n}(x, y)-3 R_{3, n}(x, y)+\frac{3}{4} R_{4, n}(x, y), \\
\vdots
\end{array}\right.
$$

Similar to the partial derivative of the variable $x$, we can show the partial derivative of the variable $y$, and from this we write the components of the matrix of derivatives $\boldsymbol{D}_{y}$ in form:

$$
\left\{\begin{array}{l}
\left(R_{m, 0}(x, y)\right)^{(1,0)}=0 \\
\left(R_{m, 1}(x, y)\right)^{(1,0)}=\frac{3}{4} R_{m, 0}(x, y)-R_{m, 1}(x, y)+\frac{1}{4} R_{m, 2}(x, y), \\
\left(R_{m, 2}(x, y)\right)^{(1,0)}=-2 R_{m, 0}(x, y)+\frac{7}{2} R_{m, 1}(x, y)-2 R_{m, 2}(x, y)+\frac{1}{2} R_{m, 3}(x, y), \\
\left(R_{m, 3}(x, y)\right)^{(1,0)}=3 R_{m, 0}(x, y)-6 R_{m, 1}(x, y)+\frac{21}{4} R_{m, 2}(x, y)-3 R_{m, 3}(x, y)+\frac{3}{4} R_{m, 4}(x, y), \\
\vdots
\end{array}\right.
$$

We assume:

$$
\left(R_{m, n}(x, y)\right)^{(1,0)}=\left(R_{m, n}(x, y)\right)^{(0,1)}=\left(R_{m, n}(x, y)\right)^{(0,0)}=0 \text { for } m>i \text { and } n>j .
$$

This assumption is based on truncating the matrices $\boldsymbol{D}_{x}$ and $\boldsymbol{D}_{y}$ to be square and the matrix multiplication become possible.

Thus, to find $\boldsymbol{R}^{(r, s)}(x, y)$ using the equalities (3.7), (3.8) as

$$
\begin{aligned}
& \mathbf{R}^{(1,0)}(x, y)=\mathbf{R}(x, y) \mathbf{D}_{x}, \\
& \mathbf{R}^{(2,0)}(x, y)=\mathbf{R}^{(1,0)}(x, y) \mathbf{D}_{x}=\left(\mathbf{R}(x, y) \mathbf{D}_{x}\right) \mathbf{D}_{x}=\mathbf{R}(x, y)\left(\mathbf{D}_{x}\right)^{2}, \\
& \boldsymbol{R}^{(3,0)}(x, y)=\boldsymbol{R}^{(2,0)}(x, y)\left(\boldsymbol{D}_{x}\right)^{2}=\boldsymbol{R}(x, y)\left(\boldsymbol{D}_{x}\right)^{3} .
\end{aligned}
$$

Then,

$$
\mathbf{R}^{(r, 0)}(x, y)=\mathbf{R}(x, y)\left(\mathbf{D}_{x}\right)^{r} .
$$

Also,

$$
\mathbf{R}^{(0, s)}(x, y)=\mathbf{R}(x, y)\left(\mathbf{D}_{x}\right)^{s},
$$

hence, from (3.9) and (3.10) we will have

$$
\mathbf{R}^{(r, s)}(x, y) \cong \mathbf{R}(x, y)\left(\mathbf{D}_{x}\right)^{r}\left(\mathbf{D}_{y}\right)^{s} .
$$

\section{Proposition 3.2}


The modified partial derivatives of order $(i, j)$-th of the row vector $\boldsymbol{R}(x, y)$ takes the following form:

$$
\begin{aligned}
\mathbf{R}^{(i, j)}(x, y)= & \mathbf{R}(x, y)\left(\mathbf{D}_{x}\right)^{i}\left(\mathbf{D}_{y}\right)^{j}+\sum_{l=0}^{j-1} \mathbf{B}_{2}^{(0,-l+j-1)}(x, y)(x, y)\left(\mathbf{D}_{y}\right)^{l}\left(\mathbf{D}_{x}\right)^{i} \\
& +\sum_{k=0}^{i-1} \mathbf{B}_{1}^{(-k+i-1,0)}(x, y)\left(\mathbf{D}_{x}\right)^{k}\left(\mathbf{D}_{y}\right)^{j} .
\end{aligned}
$$

where, $\mathbf{D}_{x}$ and $\mathbf{D}_{y}$ are given before in proposition 3.1 as (3.2) and (3.3), where $\boldsymbol{B}_{1}(x, y), \boldsymbol{B}_{2}(x, y)$ are $1 \times(m+1)(n+1)$ row vectors:

$$
\begin{aligned}
& \boldsymbol{B}_{1}(x, y)=\left[\begin{array}{llllllll}
0 & 0 & \ldots & 0 & d_{m+1, m+2} R_{m+1,0}(x, y) & 0 & 0 & \ldots
\end{array} d_{m+1, m+2} R_{m+1,1}(x, y) \ldots\right. \\
& \begin{array}{llllll}
\ldots & 0 & 0 & \ldots & \left.d_{m+1, m+2} R_{m+1, n}(x, y)\right],
\end{array} \\
& \boldsymbol{B}_{2}(x, y)=\left[\begin{array}{llllllll}
0 & 0 & \ldots & d_{n+1, n+2} R_{0, n+1} & (x, y) & 0 & 0 & \ldots d_{n+1, n+2} R_{1, n+1}(x, y) \ldots
\end{array}\right. \\
& \ldots \begin{array}{lllll} 
& 0 & 0 & \ldots & d_{n+1, n+2} R_{m, n+1} \\
& (x, y)] .
\end{array}
\end{aligned}
$$

Before we prove our proposition we note that the two summations in (3.12) are the actual terms to get the equality sign that was truncated in (3.1) by our assumption:

$$
\left(R_{m, n}(x, y)\right)^{(1,0)}=\left(R_{m, n}(x, y)\right)^{(0,1)}=\left(R_{m, n}(x, y)\right)^{(0,0)}=0 \text {, for } m>i \text { and } n>j .
$$

These added terms (two summations) will improve the obtained approximate solutions as will be shown in the numerical examples in section 6.

\section{Proof:}

The first partial derivatives of the $\boldsymbol{R}(x, y)$ can be expressed with equality sign by

$$
\mathbf{R}^{(1,0)}(x, y)=\mathbf{R}(x, y) \mathbf{D}_{x}+\mathbf{B}_{1}(x, y), \quad \mathbf{R}^{(0,1)}(x, y)=\mathbf{R}(x, y) \mathbf{D}_{y}+\mathbf{B}_{2}(x, y),
$$

consequently, to obtain the matrix $\mathbf{R}^{(i, j)}(x, y)$, using (3.15) we get:

$$
\begin{aligned}
\boldsymbol{R}^{(1,0)}(x, y) & =\boldsymbol{R}(x, y) \boldsymbol{D}_{x}+\boldsymbol{B}_{1}(x, y), \\
\boldsymbol{R}^{(2,0)}(x, y) & =\boldsymbol{R}^{(1,0)}(x, y) \boldsymbol{D}_{x}+\boldsymbol{B}_{1}^{(1,0)}(x, y)=\left(\boldsymbol{R}(x, y) \boldsymbol{D}_{x}+\boldsymbol{B}_{1}(x, y)\right) \boldsymbol{D}_{x}+\boldsymbol{B}_{1}^{(1,0)}(x, y),
\end{aligned}
$$

For this, by induction we get $i$-th partial derivative with respect to $x$ as

$$
\mathbf{R}^{(i, 0)}(x, y)=\mathbf{R}(x, y)\left(\mathbf{D}_{x}\right)^{i}+\sum_{k=0}^{i-1} \mathbf{B}_{1}^{(-k+i-1,0)}(x, y)\left(\mathbf{D}_{x}\right)^{k}, \quad i \geq 1,
$$

where

$$
\begin{aligned}
& \boldsymbol{B}_{1}^{(i, 0)}(x, y)=\left[\begin{array}{llllllllll}
0 & 0 & \ldots & d_{m+1, m+2} R_{m+1,0}^{(i, 0)}(x, y) & 0 & 0 & \ldots & d_{m+1, m+2} R_{m+1,1}^{(i, 0)}(x, y) & \ldots
\end{array}\right. \\
& \ldots \begin{array}{llllll}
\ldots & 0 & \ldots & d_{m+1, m+2} & \left.R_{m+1, n}^{(i, 0)}(x, y)\right] .
\end{array}
\end{aligned}
$$

The $j$-th derivative of the relation (3.16) with respect to the variable $y$ takes the following relation: 


$$
\begin{aligned}
\mathbf{R}^{(i, 1)}(x, y) & =\mathbf{R}^{(0,1)}(x, y)\left(\mathbf{D}_{x}\right)^{i}+\sum_{k=0}^{i-1} \mathbf{B}_{1}^{(-k+i-1,1)}(x, y)\left(\mathbf{D}_{x}\right)^{k} \\
& =\mathbf{R}(x, y) \mathbf{D}_{y}\left(\mathbf{D}_{x}\right)^{i}+\mathbf{B}_{2}(x, y)\left(\mathbf{D}_{x}\right)^{i}+\sum_{k=0}^{i-1} \mathbf{B}_{1}^{(-k+i-1,1)}(x, y)\left(\mathbf{D}_{x}\right)^{k},
\end{aligned}
$$

and

$$
\mathbf{R}^{(i, 2)}(x, y)=\mathbf{R}(x, y)\left(\mathbf{D}_{y}\right)^{2}\left(\mathbf{D}_{x}\right)^{i}+\mathbf{B}_{2}^{(0,1)}(x, y)\left(\mathbf{D}_{x}\right)^{i}+\sum_{k=0}^{i-1} \mathbf{B}_{1}^{(-k+i-1,2)}(x, y)\left(\mathbf{D}_{x}\right)^{k},
$$

finally, by induction we get $(i, j)$-th partial derivatives as

$$
\begin{aligned}
\mathbf{R}^{(i, j)}(x, y)= & \mathbf{R}(x, y)\left(\mathbf{D}_{y}\right)^{j}\left(\mathbf{D}_{x}\right)^{i}+\sum_{l=0}^{j-1} \mathbf{B}_{2}^{(0,-l+j-1)}(x, y)\left(\mathbf{D}_{y}\right)^{l}\left(\mathbf{D}_{x}\right)^{i} \\
& +\sum_{k=0}^{i-1} \mathbf{B}_{1}^{(-k+i-1, j)}(x, y)\left(\mathbf{D}_{x}\right)^{k} .
\end{aligned}
$$

Similarly, if we begin with the partial derivative of the variable $y$ then we find the $(i, j)$-th partial derivatives as

$$
\begin{aligned}
\mathbf{R}^{(i, j)}(x, y)= & \mathbf{R}(x, y)\left(\mathbf{D}_{x}\right)^{i}\left(\mathbf{D}_{y}\right)^{j}+\sum_{l=0}^{j-1} \mathbf{B}_{2}^{(i, l+j-1)}(x, y)\left(\mathbf{D}_{y}\right)^{l} \\
& +\sum_{k=0}^{i-1} \mathbf{B}_{1}^{(-k+i-1,0)}(x, y)\left(\mathbf{D}_{x}\right)^{k}\left(\mathbf{D}_{y}\right)^{j} .
\end{aligned}
$$

Then from (3.17) and (3.18) we find that

$$
\begin{aligned}
\boldsymbol{R}^{(i, j)}(x, y)= & \boldsymbol{R}(x, y)\left(\boldsymbol{D}_{x}\right)^{i}\left(\boldsymbol{D}_{y}\right)^{j}+\sum_{l=0}^{j-1} \boldsymbol{B}_{2}^{(0,-l+j-1)}(x, y)\left(\boldsymbol{D}_{y}\right)^{l}\left(\boldsymbol{D}_{x}\right)^{i} \\
& +\sum_{k=0}^{i-1} \boldsymbol{B}_{1}^{(-k+i-1,0)}(x, y)\left(\boldsymbol{D}_{x}\right)^{k}\left(\boldsymbol{D}_{y}\right)^{j}
\end{aligned}
$$

which ends the proof .

\section{Function expansions}

A well-defined function $u(x, y)$ where $(0 \leq x, y<\infty)$, can be expanded in terms of DRC functions as:

$$
u(x, y)=\sum_{r=0}^{\infty} \sum_{s=0}^{\infty} a_{r, s} R_{r, s}(x, y)
$$

where

$$
a_{r, s}=\frac{\int_{0}^{\infty} \int_{0}^{\infty} u(x, y) R_{r, s}(x, y) w(x, y) d x d y}{\int_{0}^{\infty} \int_{0}^{\infty} R_{r, s}^{2}(x, y) w(x, y) d x d y} .
$$

The expansion of $u(x, y)$ in (4.1) is truncated to $n, m$ where $n, m<\infty$ in terms of DRC functions which it be represented by:

$$
U(x, y) \cong \sum_{r=0}^{m} \sum_{s=0}^{n} a_{r, s} R_{r, s}(x, y),=\mathrm{R}(x, y) \mathrm{A},
$$


where $\boldsymbol{R}(x, y)$ is $1 \times(m+1)(n+1)$ vector with elements $R_{r, s}(x, y)$ and $\boldsymbol{A}$ is an unknown coefficient column vector are of the form:

$$
\begin{aligned}
& \mathbf{R}(x, y)=\left[\begin{array}{llllllll}
R_{0,0}(x, y) & R_{0,1}(x, y) & \ldots & R_{0, n}(x, y) & R_{1,0}(x, y) & R_{1,1}(x, y) & \ldots & R_{1, n}(x, y)
\end{array}\right.
\end{aligned}
$$

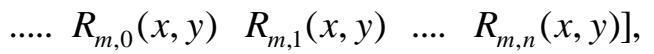

$$
\begin{aligned}
& \mathbf{A}=\left[\begin{array}{lllllllllllll}
a_{0,0} & a_{0,1} & \ldots & a_{0, n} & a_{1,0} & a_{1,1} & \ldots & a_{1, n} & \ldots & a_{m, 0} & a_{m, 1} & \ldots & a_{m, n}
\end{array}\right]^{T} .
\end{aligned}
$$

\section{Applications to PDEs}

To test the proposed definition we will use DRC technique to solve PDEs with the unknown function $\mu(x, y)$, and defined in the semi-infinite domain, which takes the form [22-23]

$$
\sum_{r=0}^{i} \sum_{s=0}^{j} p_{r, s}(x, y) \mu^{(r, s)}(x, y)=q(x, y), \quad 0 \leq(x, y)<\infty,
$$

with the non-local conditions

$$
\sum_{t=1}^{v} \sum_{r=0}^{i} \sum_{s=0}^{j} b_{r, s}^{t} \mu^{(r, s)}\left(\phi_{t}, \varphi_{t}\right)=\kappa
$$

or

$$
\sum_{t=1}^{v} \sum_{r=0}^{i} \sum_{s=0}^{j} c_{r, s}^{t}(x) \mu^{(r, s)}\left(\alpha, \theta_{t}\right)=g(x)
$$

or

$$
\sum_{t=1}^{v} \sum_{r=0}^{i} \sum_{s=0}^{j} d_{r, s}^{t}(y) \mu^{(r, s)}\left(\varepsilon_{t}, y\right)=h(y)
$$

where the $\mu^{(0,0)}(x, y)=\mu(x, y), \mu^{(r, s)}(x, y)=\frac{\partial^{r+s}}{\partial x^{r} \partial y^{s}} \mu(x, y)$ and $p_{r, s}(x, y), q(x, y), c_{r, s}^{t}(x), u(x)$, $d_{i j}^{k}(y)$ and $h(y)$ are defined on semi-infinite interval and $\phi_{t}, \varphi_{t}, \theta_{t}, \varepsilon_{t}$ are invariable $\in[0, \infty)$, especial case if one of them or more of tends to infinity. We suppose that solution $u(x, y)$ to the analytical solution $\mu(x, y)$ of Eq. (5.1) from (4.1) and its $(r, s)$ th partial order derivatives which deduced in Eq. (3.12) as:

$$
u(x, y)=\sum_{r=0}^{i} \sum_{s=0}^{j} \lambda_{r, s} R_{r, s}(x, y)=\mathbf{R}(x, y) \cdot \Lambda,
$$

and

$$
u^{(r, s)}(x, y)=\left[\begin{array}{c}
\boldsymbol{R}(x, y)\left(\boldsymbol{D}_{x}\right)^{r}\left(\boldsymbol{D}_{x}\right)^{s}+\sum_{l=0}^{j-1} \boldsymbol{B}_{2}^{(0,-l+j-1)}(x, y)\left(\boldsymbol{D}_{y}\right)^{l}\left(\boldsymbol{D}_{x}\right)^{i} \\
+\sum_{k=0}^{i-1} \boldsymbol{B}_{1}^{(-k+i-1,0)}(x, y)\left(\boldsymbol{D}_{x}\right)^{k}\left(\boldsymbol{D}_{y}\right)^{j}
\end{array}\right] \Lambda .
$$

The collocation method is used here to demonstrate the solution of eq. (5.1), the collocation points $x_{\tau}$ and $y_{\eta}$ will defined in the following: 


$$
x_{\tau}=\left(\frac{1+\cos \left(\frac{\tau \pi}{i}\right)}{1-\cos \left(\frac{\tau \pi}{i}\right)}\right), \quad y_{\eta}=\left(\frac{1+\cos \left(\frac{\eta \pi}{j}\right)}{1-\cos \left(\frac{\eta \pi}{j}\right)}\right), \quad(\tau=1, \ldots, i-1, \eta=1, \ldots, j-1),
$$

where $0 \leq x_{\tau}, y_{\eta}<\infty$.

If the variables $x$ and $y$ defined in finite interval $x \in[0, a]$ and $y \in[0, b]$ where $a$ and $b$ any positive numbers we prefer using another collocation points

$$
x_{\tau}=\frac{c}{m} \tau, \quad \tau=0,1, \ldots, m, y_{\eta}=\frac{c}{n} \eta, \eta=0,1, \ldots, n .
$$

The DRC functions specified by convergent to $x$ and $y$ even if they tend to infinity, for this, doesn't make failure in the method in unbounded domain. Then, substituting the collocation points (5.5) into (5.1) we get

$$
\left.\sum_{r=0 s=0}^{i} \sum_{r, s}^{j} p_{\tau}, y_{\eta}\right) \mu^{(r, s)}\left(x_{\tau}, y_{\eta}\right)=q\left(x_{\tau}, y_{\eta}\right),
$$

or briefly by using matrix form

$$
\sum_{r=0}^{i} \sum_{s=0}^{j} \boldsymbol{P}_{r, s} \boldsymbol{u}^{(r, s)}=\boldsymbol{Q}
$$

where $\boldsymbol{P}_{r, s}$ is the block matrix which has the diagonal elements $p_{r, s}\left(x_{\tau}, y_{\eta}\right)$ and the other elements are zeros and $Q$ is the block vector with the components $q\left(x_{\tau}, y_{\eta}\right)$. Bulging the collocation points (5.5) into partial derivatives of approximate solution $\boldsymbol{u}(x, y)$ we get

$$
\boldsymbol{u}^{(r, s)}=\left[\begin{array}{c}
u^{(r, s)}\left(x_{0}, y_{0}\right) \\
\vdots \\
u^{(r, s)}\left(x_{0}, y_{j}\right) \\
u^{(r, s)}\left(x_{1}, y_{0}\right) \\
\vdots \\
u^{(r, s)}\left(x_{1}, y_{j}\right) \\
\vdots \\
u^{(r, s)}\left(x_{i}, y_{j}\right)
\end{array}\right]=\left[\begin{array}{c}
\boldsymbol{R}\left(\boldsymbol{D}_{x}\right)^{r}\left(\boldsymbol{D}_{y}\right)^{s}+\sum_{l=0}^{j-1} \boldsymbol{B}_{2}^{(0,-l+j-1)}(x, y)\left(\boldsymbol{D}_{y}\right)^{l}\left(\boldsymbol{D}_{x}\right)^{i} \\
+\sum_{k=0}^{i-1} \boldsymbol{B}_{1}^{(-k+i-1,0)}(x, y)\left(\boldsymbol{D}_{x}\right)^{k}\left(\boldsymbol{D}_{y}\right)^{j}
\end{array}\right] \Lambda,
$$

where

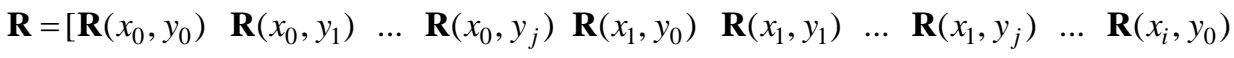

$$
\begin{aligned}
& \left.\begin{array}{llll}
\ldots \mathbf{R}\left(x_{i}, y_{1}\right) & \mathbf{R}\left(x_{i}, y_{2}\right) & \ldots & \mathbf{R}\left(x_{i}, y_{j}\right)
\end{array}\right]^{T} .
\end{aligned}
$$

Therefore, from Eq. (5.8), we get a system of the form

$$
\left(\sum_{r=0}^{i} \sum_{s=0}^{j} \boldsymbol{P}_{r, s}\left\{\begin{array}{l}
\boldsymbol{R}(x, y)\left(\boldsymbol{D}_{x}\right)^{i}\left(\boldsymbol{D}_{y}\right)^{j}+\sum_{l=0}^{j-1} \boldsymbol{B}_{2}^{(0,-l+j-1)}(x, y)\left(\boldsymbol{D}_{y}\right)^{l}\left(\boldsymbol{D}_{x}\right)^{i} \\
+\sum_{k=0}^{i-1} \boldsymbol{B}_{1}^{(-k+i-1,0)}(x, y)\left(\boldsymbol{D}_{x}\right)^{k}\left(\boldsymbol{D}_{y}\right)^{j}
\end{array}\right\}\right), \boldsymbol{Q},
$$


which is an $(i+1) \times(j+1)$ system of linear algebraic equations with $(i+1) \times(j+1) \lambda_{r, s}$ unknowns. By using the collocation points (5.5) and substitute these points in the conditions (5.2) we get the fundamental matrices of the form

$$
\begin{gathered}
\sum_{t=1}^{v} \sum_{r=0}^{i} \sum_{s=0}^{j} b_{r, s}^{t}\left\{\begin{array}{l}
\boldsymbol{R}\left(\phi_{t}, \varphi_{t}\right)\left(\boldsymbol{D}_{x}\right)^{r}\left(\boldsymbol{D}_{y}\right)^{s}+\sum_{l=0}^{j-1} \boldsymbol{B}_{2}^{(0,-l+j-1)}\left(\phi_{t}, \varphi_{t}\right)\left(\boldsymbol{D}_{y}\right)^{l}\left(\boldsymbol{D}_{x}\right)^{i} \\
+\sum_{k=0}^{i-1} \boldsymbol{B}_{1}^{(-k+i-1,0)}\left(\phi_{t}, \varphi_{t}\right)\left(\boldsymbol{D}_{x}\right)^{k}\left(\boldsymbol{D}_{y}\right)^{j}
\end{array}\right\} \Lambda=\kappa, \\
\sum_{t=1}^{v} \sum_{r=0}^{i} \sum_{s=0}^{j} c_{r, s}^{t}\left(x_{\tau}\right)\left\{\begin{array}{l}
\boldsymbol{R}\left(x_{\tau}, \theta_{t}\right)\left(\boldsymbol{D}_{x}\right)^{r}\left(\boldsymbol{D}_{y}\right)^{s}+\sum_{l=0}^{j-1} \boldsymbol{B}_{2}^{(0,-l+j-1)}\left(x_{\tau}, \theta_{t}\right)\left(\boldsymbol{D}_{y}\right)^{l}\left(\boldsymbol{D}_{x}\right)^{i} \\
+\sum_{k=0}^{i-1} \boldsymbol{B}_{1}^{(-k+i-1,0)}\left(x_{\tau}, \theta_{t}\right)\left(\boldsymbol{D}_{x}\right)^{k}\left(\boldsymbol{D}_{y}\right)^{j}
\end{array}\right\} \Lambda=g\left(x_{\tau}\right), \\
\sum_{t=1}^{v} \sum_{r=0}^{i} \sum_{s=0}^{s} d_{r, s}^{t}\left(y_{\eta}\right)\left\{\begin{array}{l}
\boldsymbol{R}\left(\varepsilon_{t}, y_{\eta}\right)\left(\boldsymbol{D}_{x}\right)^{r}\left(\boldsymbol{D}_{y}\right)^{s}+\sum_{l=0}^{j-1} \boldsymbol{B}_{2}^{(0,-l+j-1)}\left(\varepsilon_{t}, y_{\eta}\right)\left(\boldsymbol{D}_{y}\right)^{l}\left(\boldsymbol{D}_{x}\right)^{i} \\
+\sum_{k=0}^{i-1} \boldsymbol{B}_{1}^{(-k+i-1,0)}\left(\varepsilon_{t}, y_{\eta}\right)\left(\boldsymbol{D}_{x}\right)^{k}\left(\boldsymbol{D}_{y}\right)^{j}
\end{array}\right\} \Lambda=h\left(y_{\eta}\right) .
\end{gathered}
$$

The fundamental matrix (5.9) for eq. (4.1) is transformed to a system of $(i+1) \times(j+1)$ algebraic equations for the $(i+1) \times(j+1)$ unknown $\mathrm{RC}$ coefficients

$$
\left[\begin{array}{lllllllllll}
\lambda_{00} & \lambda_{01} \ldots & \lambda_{0 j} & \lambda_{10} & \lambda_{11} \ldots & \lambda_{1 j} & \ldots & \lambda_{i 0} & \lambda_{i 1} & \ldots & \lambda_{i j}
\end{array}\right]
$$

We can write the matrix (4.8) as:

$$
\boldsymbol{Y} \Lambda=\boldsymbol{Q} \quad \text { or } \quad[\boldsymbol{Y} ; \boldsymbol{Q}] \text {. }
$$

Also, we write the fundamental matrix of the conditions from (4.10) as follows

$$
\boldsymbol{X} \Lambda=\boldsymbol{G}, \text { or }[\boldsymbol{X} ; \boldsymbol{G}],
$$

where $\boldsymbol{X}$ is a $h \times(i+1)(j+1)$ matrix and $\boldsymbol{G}$ is a $h \times 1$ matrix, so that $h$ is the rank of the all row matrices as in (5.11) belong to the given conditions.

Thus, systems (5.11) and (5.12) will be expressed as follows:

$$
\mathbf{Y}^{*} \Lambda=\mathbf{Q}^{*} \text {, or }\left\lfloor\mathbf{Y}^{*} ; \mathbf{Q}^{*}\right\rfloor \text {. }
$$

Hence, the equations (5.13) can be compacted by putting the vectors (5.12) on conditions to the equations (5.11). We use the generalized inverse [20] of $\boldsymbol{Y}^{*}$ for solving equations (5.13), and then we get the unknown $\lambda_{r, s}$ from the following:

$$
\Lambda=\operatorname{geninv}\left(\boldsymbol{Y}^{*}\right) \cdot \boldsymbol{Q}^{*}
$$

In addition, (5.9) or (5.11) is derived a modified scheme, also, if $\boldsymbol{B}_{1}(x, y)$ and $\boldsymbol{B}_{2}(x, y)$ are vanish then the regular scheme applied, and they will removed from all steps (5.8) - (5.10).

\section{Numerical examples}


In this section, we apply the DRC collocation method is on some test examples to obtain its applicability and validity. All numerical examples are computed on the computer by MATHEMATICA 7.0.

\section{Problem 1}

Consider the PDEs of order two

$$
\frac{1}{x+1} \mu^{(0,2)}(x, y)+\frac{1}{(x+1)(y+1)} \mu^{(1,1)}(x, y)=\frac{-4\left(x^{2}-2\right)}{(x+1)^{3}(y+1)^{3}}, \quad x, y \in[0, \infty),
$$

and the conditions for this test example are:

$$
\mu(x, y)=1 \text { at } x \rightarrow \infty \text { and at } y \rightarrow \infty, \mu(0,0)=1,
$$

the fundamental matrix takes the form

$$
\left\{\begin{array}{l}
\boldsymbol{P}_{0,2}\left[\boldsymbol{R}\left(\boldsymbol{D}_{y}\right)^{2}+\boldsymbol{B}_{2}^{(0,1)}(x, y)\left(\boldsymbol{D}_{y}\right)^{0}+\boldsymbol{B}_{2}^{(0,0)}(x, y)\left(\boldsymbol{D}_{y}\right)^{1}\right] \\
+\boldsymbol{P}_{1,1}\left[\boldsymbol{R}\left(\boldsymbol{D}_{y}\right)^{1}\left(\boldsymbol{D}_{y}\right)^{1}+\boldsymbol{B}_{2}^{(0,0)}(x, y)\left(\boldsymbol{D}_{x}\right)^{1}+\boldsymbol{B}_{1}^{(0,0)}(x, y)\left(\boldsymbol{D}_{y}\right)^{1}\right]
\end{array}\right\} \Lambda=\boldsymbol{Q},
$$

Consider $i=j=4$, where, the approximate solution has the form

$$
u(x, y)=\lambda_{0,0} R_{0,0}(x, y)+\lambda_{0,1} R_{0,1}(x, y)+\cdots+\lambda_{4,4} R_{4,4}(x, y),
$$

For this, we compute the solve of the augmented matrix of the fundamental relation and conditions, we will get the unknown coefficients of RC functions as,

$$
\begin{aligned}
& \lambda_{0,0}=\lambda_{0,1}=\ldots=\lambda_{0,4}=0, \\
& \lambda_{1,0}=0, \lambda_{1,1}=1, \lambda_{1,2}=0, \ldots=\lambda_{1,4}=0, \\
& \lambda_{2,0}=0, \lambda_{2,1}=0, \ldots=\lambda_{2,4}=0 \\
& \lambda_{3,0}=0, \lambda_{3,1}=0, \ldots=\lambda_{3,4}=0 \\
& \lambda_{4,0}=\lambda_{4,1}=\ldots=\lambda_{4,4}=0
\end{aligned}
$$

Then, using the relation (2.2) we get the exact solution $\mu(x, y)=\frac{x y-x-y+1}{(x+1)(y+1)}$, of problem (6.1).

Problem 2 Consider Poisson's equation

$$
\mu_{x x}+\mu_{y y}=-2 e^{-x-y}
$$

with the Dirichlet boundary conditions

$$
\mu(0, y)=e^{-y}, \quad \mu(x, 0)=e^{-x} \quad \mu(1, y)=e^{-1-y}, \quad \mu(x, 1)=e^{-x-1} \quad \text { where } \quad 0 \leq x, y \leq 1 .
$$

The introduced two proposed techniques are using to solve Poisson's equation (6.2). We see in table 1 the comparison between the exact (which is $\mu(x, y)=e^{-x-y}$ ) and approximate solutions tabulated (the numerical results for $i=j=8$ ). In addition, table 2 compares the $L_{2}, L_{\infty}$ error norms of the modified and regular schemes with different values of $i, j$. In addition, we illustrate in figures 1 and 2 the absolute errors function for the two schemes at $i=j=8$. Previous discussion shows that the grater $i, j$ give good accuracy and the present technique which used the improved scheme gives us accuracy better than regular scheme. 
Table. 1 Absolute errors for various values of $i, j$ where $\mathrm{y}=0.1$ and $x$ takes various values

\begin{tabular}{|c|c|c|c|c|c|}
\hline$x$ & Exact solution & $\begin{array}{c}\text { Regular } \\
\text { scheme } \\
\boldsymbol{i = j = 8}\end{array}$ & $\begin{array}{c}\text { Absolut } \\
\text { error }\end{array}$ & $\begin{array}{c}\text { Modified } \\
\text { scheme } \boldsymbol{i = j = 8}\end{array}$ & Absolut error \\
\hline 0 & 0.9048374180 & 0.9048371375 & $2.804 \times 10^{-7}$ & 0.9048374183 & $3.651 \times 10^{-10}$ \\
\hline 0.1 & 0.8187307530 & 0.8187312589 & $5.058 \times 10^{-7}$ & 0.8187307591 & $6.152 \times 10^{-9}$ \\
\hline 0.2 & 0.7408182206 & 0.7408204798 & $2.259 \times 10^{-6}$ & 0.7408182238 & $3.211 \times 10^{-9}$ \\
\hline 0.3 & 0.6703200460 & 0.6703153776 & $4.668 \times 10^{-6}$ & 0.6703200391 & $6.971 \times 10^{-9}$ \\
\hline 0.4 & 0.6065306597 & 0.6065197858 & $1.087 \times 10^{-5}$ & 0.6065306485 & $1.127 \times 10^{-8}$ \\
\hline 0.5 & 0.5488011924 & 0.5488011924 & $1.044 \times 10^{-5}$ & 0.5488019874 & $7.952 \times 10^{-7}$ \\
\hline 0.6 & 0.4965853037 & 0.4965803342 & $4.96 \times 10^{-6}$ & 0.4965853166 & $1.296 \times 10^{-8}$ \\
\hline 0.7 & 0.4493289641 & 0.4493302679 & $1.303 \times 10^{-6}$ & 0.4493288701 & $9.478 \times 10^{-8}$ \\
\hline 0.8 & 0.4065696597 & 0.4065746321 & $4.972 \times 10^{-6}$ & 0.4065696389 & $2.087 \times 10^{-8}$ \\
\hline 0.9 & 0.3678794411 & 0.3678840147 & $4.573 \times 10^{-6}$ & 0.3678794401 & $1.725 \times 10^{-9}$ \\
\hline 1 & 0.3328710836 & 0.3328715031 & $4.194 \times 10^{-7}$ & 0.3328710844 & $8.791 \times 10^{-10}$ \\
\hline
\end{tabular}

Table. 2 Comparing the $L_{2}$ and $L_{\infty}$ error norms

\begin{tabular}{|c|c|c|c|c|}
\hline & $\begin{array}{c}L_{2} \text { regular } \\
\text { scheme }\end{array}$ & $\begin{array}{c}L_{2} \text { modified } \\
\text { scheme }\end{array}$ & $\begin{array}{c}L_{\infty} \text { Regular } \\
\text { scheme }\end{array}$ & $\begin{array}{c}L_{\infty} \text { modified } \\
\text { scheme }\end{array}$ \\
\hline$i=j=8$ & $9.57079 \times 10^{-9}$ & $7.8361 \times 10^{-12}$ & $1.29247 \times 10^{-5}$ & $1.4324 \times 10-6$ \\
\hline$i=j=10$ & $4.37445 \times 10-8$ & $3.2145 \times 10^{-13}$ & $1.77424 \times 10^{-5}$ & $4.3217 \times 10^{-7}$ \\
\hline
\end{tabular}

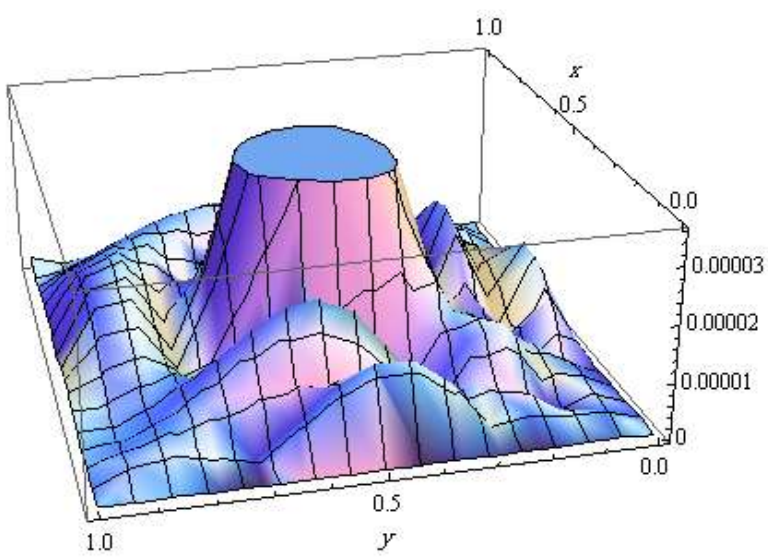

Figure.1 Error function for regular scheme at $i=j=8$

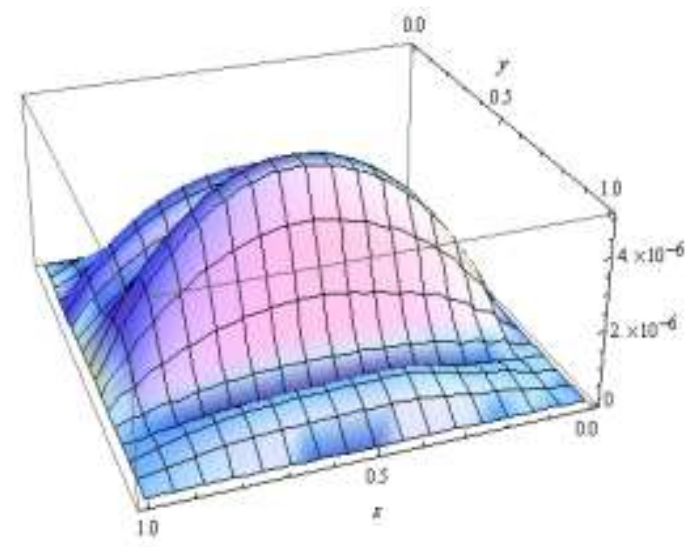

Figure.2 Error function for improved scheme at $i=j=10$ 


\section{CONCLUSIONS}

In this work, a collocation technique for linear PDEs defined on semi-infinite domain under mixed conditions with variable coefficients is proposed. The technique is based on the approximating the solution function by the truncated DRC series. The definitions of the partial derivatives of DRC functions are introduced in a regular and an improved form. The regular and improved definitions generate two schemes with the collocation method. The PDEs and conditions are transformed to block matrix equations. This matrix equation is a system of linear algebraic equations with the unknown RC coefficients. Test examples are used to demonstrate the applicability, effectiveness and the accuracy of the proposed techniques. Also, the numerical results obtained that the improved scheme gives better accuracy than the regular scheme.

\section{REFERENCES}

[1] Ayşegül Akyüz, Mehmet Sezer, Chebyshev polynomial solutions of systems of high-order linear differential equations with variable coefficients, Applied Mathematics and Computation 144 (2003) 237-247.

[2] J.C. Mason, D.C. Handscomb, Chebyshev polynomials, CRC Press, Boca Raton, 2003.

[3] G. Yuksel, M. Gulsu and M. Sezer, A Chebyshev Polynomial Approach for Higher - Order Linear Fredholm-Volterra Integro-Differential Equations, Gazi University Journal of Science, 2012,25(2), 393 401.

[4] B. Kafasha, A. Delavarkhalafi, S.M. Karbassi, Application of Chebyshev polynomials to derive efficient algorithms for the solution of optimal control problems, Scientia Iranica, Vol. 19, Issue 3, June 2012, Pages 795-805.

[5] Kamal R. Raslan KR, Khalid K. Ali, Mohammad A. Abd El Salam, \& Emad M. Mohamed, Spectral Tau method for solving general fractional order differential equations with linear functional argument. Journal of the Egyptian Mathematical Society 2019; 27(1): 33.

[6] Khalid K. Ali, Mohammad A. Abd El Salam \& Emad M. Mohamed (2019).Chebyshev operational matrix for solving fractional order delay-differential equations using spectral collocation method. Arab jornal of Basic Applied sciences University of Bahrain, 26 (1), 342-353.

[7] N. K. Basu, On double Chebyshev series approximation, SIAM. J. Numer. Anal., 10(3) (1973), 496-505.

[8] A. B. Koc and A. Kurnaz, A new kind of double Chebyshev polynomial approximation on unbounded domains, Boundary Value Problems, 1 (2013), 1687-2770.

[9] J.P. Boyd, Orthogonal rational functions on a semi-infinite interval, J. Comput. Phys. 70, 63-88, 1987

[10] Ramadan, M., Raslan, K., Hadhoud, A. and Nassar, M. A Rational Chebyshev Functions Approach for Fredholm-VolterraIntegro-Differential Equations.Comput. Methods Differ. Equ. 2015, 3, 284-297.

[11] Ramadan, M.; Raslan, K.; Nassar, M. An approximate solution of systems of high-order linear differential equations with variable coefficients by means of a rational Chebyshev collocation method.Electron. J. Math. Anal. Appl. 2016, 4, 42-48.

[12] Ramadan, M.; Raslan, K.; Nassar, M. Numerical Solution of System of Higher Order Linear Ordinary Differential Equations with Variable Coeficients Using Two Proposed Schemes for Rational Chebyshev Functions. Glob. J. Math. 2015, 3, 322-327.

[13] Ramadan, M., Raslan, K., Nassar, M. Numerical Solution of high-order linear integro-differential equations with Variable Coeficients using two proposed schemes for Rational Chebyshev functions. New Trends Math. Sci. 2016, 4, 22-35.

[14] Ramadan, M.; Raslan, K.; Hadhoud, A.; Nassar, M. Rational Chebyshev functions with new collocation points in semi-infinite domains for solving higher-order linear ordinary differential equations. J. Adv. Math. 2015, 11, 5403-5410.

[15] Ramadan, M.; Raslan, K.; Nassar, M. An approximate analytical solution of higher-order linear differential equations with variable coefficients using improved rational Chebyshev collocation method. Appl. Comput. Math. 2014, 3, 315-322. 
[16] M. A. Ramadan and Mohamed. A. Abd El salam, Spectral collocation method for solving continuous population models for single and interacting species by means of exponential Chebyshev approximation, International Journal of Biomathematics, 11:8, 1850109, (2018).

[17] M. A. Ramadan and Mohamed. A. Abd El salam, Solving systems of ordinary differential equations in unbounded domains by exponential Chebyshev collocation method, Jornal of abstract and computational mathematics, 1:1, 33-46, (2016).

[18] Parand K, Razzaghi M. Rational Chebyshev tau method for solving Volterra's population model. Applied Mathematics and Computation. 2004 Feb 22;149(3):893-900.

[19] Parand K, Delafkar Z, Baharifard F. Rational Chebyshev Tau method for solving natural convection of Darcian fluid about a vertical full cone embedded in porous media whit a prescribed wall temperature. World Academy of Science, Engineering and Technology, 2011 Aug. 24; 5 (8):1186-1191.

[20] Sezer M, Gülsu M, Tanay B. Rational Chebyshev collocation method for solving higher-order linear ordinary differential equations. Numerical Methods for Partial Differential Equations. 2011 Sep; 27(5):1130-42.

[21] M. A. Ramadan, K. R. Raslan, T. S. El Danaf and M. A. El Salam, Modified exponential Chebyshev operational matrices of derivatives for solving high-order partial differential equations in unbounded domains, Electronic Journal of Mathematical Analysis and Applications 5(2) (2017) 229-241.

[22] M. A. Ramadan, K. R. Raslan, T. S. El Danaf and M. A. El Salam, Solving high-order partial differential equations in unbounded domains by means of double exponential second kind Chebyshev approximation, Computational Methods for Differential Equations 3(3) (2015) 147-162

[23] Akyüz Dascioglu A. Chebyshev polynomial approximation for high order partial differential equations with complicated conditions. Numerical Methods for Partial Differential Equations: An International Journal. 2009 May;25(3):610-21.

\section{اطروحة معدلة جديدة لحل معادلات تفاضلية جزئية ذات معاملات متغيرة فى مجال شبة دئة

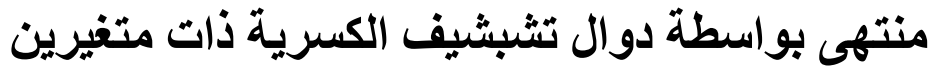

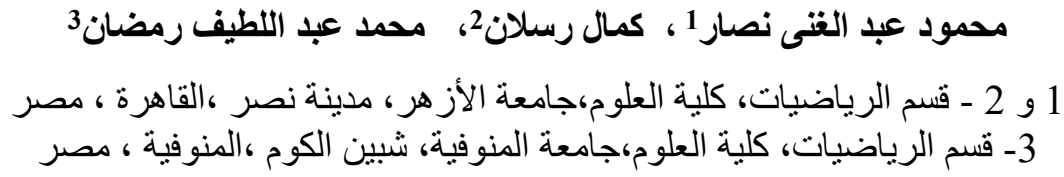

